\title{
HEISENBERG Y BOHR EN LA MIRA DE FRAYN Y VOLPI
}

\author{
Alí Víquez, Jiménez.
}

\section{(c)}

Esta obra está bajo una licencia Creative Commons

Reconocimiento-No Comercial-Sin Obra Derivada 



\title{
HEISENBERG Y BOHR EN LA MIRA DE FRAYN Y VOLPI
}

\section{HEISENBERG AND BOHR IN THE SIGHT OF FRAYN AND VOLPI}

\section{Alí Víquez Jiménez}

\begin{abstract}
RESUMEN
Este artículo compara las versiones literarias que, de las relaciones entre Werner Heisenberg y Niels Bohr, presentan Michael Frayn (Copenhague, pieza teatral de 1998) y Jorge Volpi (En busca de Klingsor, novela de 1999). En particular, se detiene en la controversia del encuentro de 1941, y asimismo en la lectura que de los problemas específicamente científicos se hace en dichos textos literarios.

Palabras clave: física y literatura, ciencia y literatura, literatura comparada, literatura mexicana contemporánea, literatura inglesa contemporánea.
\end{abstract}

\begin{abstract}
This article compares the literary interpretations of the relationship between Werner Heisenberg and Niels Bohr as presented in Michael Frayn's Copenhague (theatrical work, 1998) and Jorge Volpi's En busca de Klingsor (novel, 1999). Particular emphasis is placed on the controversial encounter between these two scientists in 1941, and likewise on the literary rendition of specifically scientific issues in the two texts under consideration.

Key words: physics and literature, science and literature, comparative literature, contemporary Mexican literature, contemporary British literature.
\end{abstract}

\section{Introducción}

Tanto Jorge Volpi como Michael Frayn se han ocupado de recrear literariamente el episodio histórico que el primero califica como "[...] el ríspido encuentro de Bohr y Heisenberg en la capital danesa en septiembre de 1941." (Volpi, 2000, p. 441) Lo hicieron casi simultáneamente: Volpi le daba los toques finales a En busca de Klingsor cuando, en mayo de 1998, se estrena en Londres Copenhague. El primero en advertir esta coincidencia fue nada menos que Carlos Fuentes, según lo establece el propio Volpi (2000, p. 441).

M.L. Alí Víquez Jiménez. Universidad de Costa Rica. Profesor de la Escuela de Filología, Lingüística y Literatura. Costa Rica.

Correo electrónico: aviquez@yahoo.com

Recepción: 14- 01- 2014

Aceptación: 19- 02- 2014 
Dos personajes históricos, grandes genios de la física contemporánea: a Heisenberg se le debe el principio de incertidumbre; a Bohr, la complementariedad; de ambos procede la Interpretación de Copenhague. Y estos no son sino los logros mayores de dos carreras plenas de éxitos, que se desarrollaron gracias a un trabajo en una parte colaborativo y en otra parte individual, desde los años veinte. Un encuentro en 1941 asimismo histórico, dado en las difíciles circunstancias de la ocupación nazi de Dinamarca: Bohr era danés y Heisenberg alemán. Acontecimiento al que tal vez habría que llamar (para ir adentrándonos en la jerga de la física de partículas a la cual estos científicos se supieron dedicar) un choque, una colisión. Como si ellos mismos hubieran sido proyectiles diminutos lanzados en un acelerador lineal el uno contra el otro, Heisenberg y Bohr se golpean y acaso se despedazan, al menos por un breve momento de sus vidas. Sobre el particular existe bibliografía no literaria, que tanto Michael Frayn como Jorge Volpi consultaron (Frayn, 2000, pp. 95-97; Volpi, 2000, pp. 441-442).

Mi interés en esta ocasión es comparar en detalle las dos versiones literarias elaboradas por Michael Frayn y Jorge Volpi en torno a la relación entre Heisenberg y Bohr, desde los años veinte hasta los cuarenta, tomando en cuenta los aportes que estos dos últimos hicieron a la ciencia. ${ }^{1}$ No procuro aclarar episodios históricos o temas científicos; más bien, comparar dos lecturas literarias de un prolongado encuentro que tienen justificación no en su relación con un referente sin remedio perdido sino en su propuesta estética; es decir, en última instancia, dos versiones que contribuyen a crear lo que todo texto literario crea: modelos ficcionales del mundo. Estos modelos no son gratuitos, arbitrarios o meramente fantasiosos; pero su relación con la realidad se construye no en los términos referenciales del reportaje o la historia (por cierto, también convencionales y dudosos hasta puntos que no viene al caso pormenorizar), sino en los términos ficcionales propios de la literatura. Para esta, no se trata tanto de decir la verdad cuanto de hurgar en ella por la vía preferida de la ficción: ser una verdad que miente. La frase de Eco (1981) resulta especialmente válida cuando la literatura se relaciona abiertamente, como en los casos presentes, con personajes y documentos históricos. ${ }^{2}$

Al contrario de lo que ocurre con el colisionador de partículas en Ginebra (el mayor de todos, hasta la fecha), ${ }^{3}$ no me será necesario aumentar la velocidad de los textos para comparar los resultados del choque. Pero este choque existe, de hecho, por la simple razón de que son textos diferentes, que no pueden sino separarse acaso en parte del modo en que se separan los componentes de las partículas cuando entran violentamente en contacto. El choque está dado por el hecho mismo de que siempre hay un algo violento en una separación, aunque sea esta parcial (y creo que es el caso; de aquí el interés de la comparación, que sería aburrida si no hubiera más que coincidencia y sobrante si no hubiera más que separación). Yo procederé más bien con parsimonia; llevaré los textos lentamente hacia los puntos en que se encuentran y trataré de hacer el recuento de lo que se expresa en su separación. Uno de los errores que me parecen más comunes a la hora de explicar a los legos la mecánica cuántica (hora era esta de mencionarla, aunque no lo hago sin el temor de quien incursiona en un campo en donde lo pueden llamar intruso) es el de tratar de asimilar los acontecimientos microscópicos a los macroscópicos, dado que sobre estos hay mejor entendimiento general o al menos una intuición al respecto que podría colaborar. Pero ello es como tratar de asimilar dos órdenes que deben mantenerse separados si se los quiere comprender adecuadamente, al menos en un inicio. (No se me pasa que una de las grandes metas de la física contemporánea es la unificación de la relatividad general con la mecánica cuántica; es decir, justamente, la creación de una teoría que permita comprender los fenómenos macroscópicos y los microscópicos a la vez, pero aquí hablo de la forma en que hay que comenzar 
a entender la mecánica cuántica: desprendiéndose de los prejuicios que nos proporcionan nuestras intuiciones sobre el mundo mayúsculo.) Me esforzaré por no cometer un error análogo, en el sentido inverso, al que me veo tentado: me es imposible tratar con las partículas elementales de los textos literarios; estos solamente conocen una cierta "macroexistencia". Debe lidiarse, al menos, con sus "moléculas", entendidas estas como las partículas más pequeñas que existen en la naturaleza: sí hay más elementos todavía más chicos, pero no corresponden a la organización natural del mundo. ¿Cuáles son las "moléculas" del mundo literario? Es una pregunta que sólo puede responderse desde la práctica de la lectura: no existe una definición apriorística satisfactoria, y deberá el crítico darse sus propios segmentos. (Véase, pero solamente como una referencia a la discusión teórica, la introducción de Barthes [1987] a S/Z. Hago la salvedad de que la segmentación con la que Barthes trabaja el texto de Balzac en esa ocasión siempre me ha parecido excesiva o, si se quiere continuar con el símil propuesto, yo creo que Barthes pasa allí de lo molecular a lo atómico).

Para terminar esta nota introductoria, deseo hacer patente mi agradecimiento al Dr. Manuel Ortega, catedrático de la Escuela de Física de la Universidad de Costa Rica, por su gentil revisión de los aspectos propiamente ligados a la física que abordo en este artículo. De más está decir que la responsabilidad por los errores al tratar una disciplina que no es mi especialidad sigue siendo toda mía.

\section{Los textos policiacos}

En Copenhague participan únicamente tres personajes: Heisenberg, Bohr y la esposa de este, Margrethe. Es ella quien dice la primera frase del texto dramático, una interrogante que nos da el propósito de toda la obra: responder a la pregunta de por qué vino Heisenberg a Copenhague en 1941. Es una pregunta que ha intrigado a los historiadores de la ciencia y en torno a ella se organiza el drama de Michael Frayn. Estamos entonces en presencia de lo que casi es un drama policial, con una complicación adicional: no se trata aquí tan sólo de resolver quién cometió un crimen, sino de procurar responder si hubo, en primer lugar, un crimen y, en caso de haberse dado, por qué lo habría cometido quien lo hizo. La interrogante tradicional del género policiaco (¿quién es el culpable?) se convierte en una más intrigante quizá: hay que comenzar por preguntarse si se ha dado una falta; luego, hay que entender por qué se dio, si fuera el caso. Quién la cometió parece estar claro al principio: de haber existido un crimen, el culpable es Heisenberg, el alemán acaso fiel a su gobierno nazi, acaso capaz incluso de buscar la colaboración de Bohr para proveer a Hitler de una bomba atómica. Ahora bien, es Margrethe quien jamás duda de que el único culpable, de haberlo, debe ser Heisenberg, ya que considera a su marido incapaz de nada malo, pero ya veremos que a medida que el drama avanza, y sobre todo hacia su término, también aparece la interrogante de si Bohr se podría considerar culpable. Después de todo, este participó en el proyecto de Los Álamos, donde finalmente se construyó la bomba atómica que jamás tuvieron los alemanes. En el peor de los escenarios, Heisenberg fue culpable de haber intentado crear la bomba para los nazis, mientras que Bohr formó parte del equipo que efectivamente construyó la bomba para matar en el Japón. Por supuesto, hay una diferencia entre colaborar con Hitler y colaborar con los aliados; pero también hay una diferencia entre tratar de obtener un arma y apretar el gatillo para que de hecho mueran una enorme cantidad de civiles inocentes. ${ }^{4}$ De manera que la interrogante tradicional del género policiaco (¿quién es el culpable?) también está puesta sobre la mesa. 
La novela de Volpi se inscribe abiertamente en el género policiaco. Desde el título, el planteamiento está dado: se trata de buscar a Klingsor, nombre de un personaje de la ópera Parsifal, de Wagner, detrás del cual se esconde el cerebro a cargo de la investigación científica durante el régimen nazi. Un físico y militar estadounidense, llamado significativamente Francis Bacon, desarrolla la investigación al término de la guerra, con el objetivo de sentar las responsabilidades de quienes colaboraron con Hitler. Pero la narración corre a cargo de un narrador que alterna como testigo y como protagonista y que eventualmente se considerará como uno de los sospechosos de ser Klingsor: el matemático Gustav Links. Ellos dos son los únicos personajes vinculados a la ciencia en la novela que no cuentan con referentes históricos: todos los otros científicos que aquí aparecen son elaboraciones hechas sobre la base de la investigación sobre ciencia realizada por Volpi, los grandes físicos y matemáticos de la época: Einstein, Planck, Bohr, Heisenberg, Schrödinger, Stark, Gödel, Von Neumann. De esta lista se extraen los nombres de quienes podrían ser Klingsor: Heisenberg y Stark como sospechosos principales (junto a Links, al final); algún otro como Planck parece excluido. (Si bien hay que tomar en cuenta que una de las estrategias de más peso en el género policiaco es culpar a quien menos ha levantado sospechas; no obstante, y dado precisamente el hecho de que Volpi se vale de personajes que cuentan con un referente histórico, la verosimilitud de la narración se vería seriamente comprometida si se le hubiera ocurrido, por ejemplo, culpar a Einstein de haber asesorado a Hitler para la consecución de la bomba atómica). ${ }^{5}$ El personaje de Francis Bacon no pretende relacionarse con su referente histórico por razones en primer lugar cronológicas y obvias: si el mundo ficcional de la narración se ubica alrededor de la segunda guerra mundial, habría que dar cabida a una licencia fantástica para que un filósofo y científico de la Inglaterra isabelina apareciera allí, y no es el caso. De hecho, habiendo destacado como niño prodigio en las matemáticas, el personaje de Francis Bacon de la novela - un nada misterioso miembro de la clase media estadounidense- es objeto de burlas por parte de alguno de sus maestros, debido precisamente a que lleva ese nombre. Pero no es posible, por lo demás, pasar por alto la alusión efectuada por el hecho de que se llame así: el personaje de la novela se construye como el portador de un nombre que lo mueve a investigar: Bacon (el otro, el histórico) es el padre del empirismo, que promueve un saber resultado de la observación y nunca de los prejuicios, y que sólo se valida en la experiencia. Es lo que el Francis Bacon de la novela debe hacer: observar para descubrir quién es Klingsor. ${ }^{6}$

Los dos textos, pues, se plantean como elaboraciones en torno a la resolución de enigmas. En Copenhague, el enigma primordial gira en torno al encuentro Heisenberg-Bohr de 1941; por su parte, En busca de Klingsor no se centra en las preguntas sobre este episodio, sino que se las hace como parte de una investigación sobre un problema más general, el de cómo se organizaba la física alemana durante la guerra. Para llegar a este punto en 1941, hay que remontarse al momento de oro en que se gestó la mecánica cuántica, el gran marco científico que unió a Heisenberg y Bohr. Ambos textos nos dan sus versiones al respecto de esa gestación durante los años veinte. Continuaré comparándolas.

\section{Las gestaciones de la mecánica cuántica: incertidumbre,complementariedad e interpretación probabilística de la función de onda}

El punto de partida ronda una época en la que Alemania, perdedora en la Primera Guerra Mundial, produce sin embargo una gran cantidad de físicos interesantísimos. Pero no sólo Alemania; recuérdese que Bohr es danés, por ejemplo, y Schrödinger vienés. A diferencia 
de la relatividad, obra del genio individual de Einstein, la mecánica cuántica será el resultado de una creación colectiva en la que, por supuesto, participaron Bohr y Heisenberg, así como otros más (Born, Pauli y Schrödinger, entre otros). El aporte fundamental inicial de Heisenberg lo constituye el principio de incertidumbre: "Cuanto más precisa sea la medida de la posición de un objeto, más incierta será su velocidad. Y viceversa, cuanto más precisa sea la velocidad de un objeto, más incierta será su posición.” (Rosenblum y Kuttner, 2011, p. 131) No es una imprecisión dada por las limitaciones de los instrumentos de medida; se trata de algo que compromete seriamente la forma como la realidad existe: de manera incierta. Así lo plantean Hawkins y Mlodinow:

\begin{abstract}
Según la física cuántica, sea cual sea nuestra capacidad de obtener información o nuestra capacidad de cálculo, no podemos predecir con certidumbre los resultados de los procesos físicos porque no están determinados con certidumbre. En lugar de ello, dado el estado inicial de un sistema la naturaleza determina su estado futuro mediante un proceso fundamentalmente incierto. En otras palabras, la naturaleza no dicta el resultado de cada proceso o experimento ni siquiera en las situaciones más simples. Más bien, permite un número de eventualidades diversas, cada una de ellas con una cierta probabilidad de ser realizada. Es, parafraseando a Einstein, como si Dios lanzara los dados antes de decidir el resultado de cada proceso físico. (Hawkins y Mlodinow, 2010, pp. 82-83)
\end{abstract}

Se sabe que Einstein mismo se sintió repugnado ante tal propuesta, la cual incluso hoy encuentra impugnadores, si bien estos son minoría. Las consecuencias, según como este principio de incertidumbre se interprete, son tales que físicos como Rosenblum y Kuttner sostienen que, al menos alguna vez, la opinión de Heisenberg fue que, en última instancia, los objetos microscópicos mismos no son cosas reales. De hecho, estos científicos citan a un Heisenberg que se aleja muchísimo de considerar "realista" a la mecánica cuántica:

\footnotetext{
[...] en los experimentos sobre sucesos atómicos tenemos que tratar con cosas y con hechos, con fenómenos que sean tan reales como cualquier fenómeno de la vida cotidiana. Pero los átomos o las partículas elementales en sí no son reales; constituyen un mundo de potencialidades o posibilidades y no de cosas o hechos. (Rosenblum y Kuttner, 2011, p. 128)
}

Por ello, no es de extrañar la actitud de Einstein: la mecánica cuántica es altamente contraintuitiva. Alguna vez declaró que, viendo lo que la física de Bohr y compañía había pergeñado, hubiera preferido ser panadero antes que físico. El propio Bohr dijo: "Si alguien dice que puede pensar en la mecánica cuántica sin sentir vértigo, entonces es que no ha entendido nada de nada." (citado por Gell-Mann, 2007, p. 185).

El principio de incertidumbre convierte a la física en un área del saber que lidia, básicamente, con probabilidades. Según Hawkins y Mlodinow:

\footnotetext{
Puede parecer que la física cuántica mine la idea de que la naturaleza está regida por leyes, pero no es ése el caso, sino que nos lleva a aceptar una nueva forma de determinismo: dado el estado de un sistema en un cierto momento, las leyes de la naturaleza determinan las probabilidades de los diversos futuros y pasados en lugar de determinar con certeza un futuro y un pasado. Aunque esto resulta desagradable para algunos, los científicos debemos aceptar teorías que concuerden con los experimentos y no con nuestras nociones preconcebidas. (Hawkins y Mlodinow, 2010, p. 83)
}

Además, ellos insisten en las diferencias fundamentales existentes entre las probabilidades en la "vida corriente" (que pueden describirse según la física newtoniana) y en la física cuántica. Pues las probabilidades de que, por ejemplo, un proyectil se dirija a un punto dado pueden conocerse con entera precisión si se calculan exactamente variables de la física clásica como ángulo, rotación, velocidad, etcétera. Si se conoce todo el espectro de los factores determinantes, se podrá predecir con entera certeza el punto con el que el proyectil 
hará contacto; incluso, se podrá planear que lo haga, y el momento en que lo hará. En cambio, en teoría cuántica las probabilidades funcionan de otra forma. Si impulsamos una partícula microscópica,

[...] por grandes que sean nuestras habilidades y conocimientos no podremos predecir con exactitud dónde aterrizará. Pero si repetimos muchas veces dicho experimento, los datos que obtengamos reflejarán la posibilidad de hallarlo en diversas posiciones [...] (Hawkins y Mlodinow, 2010, p. 84)

En la misma línea de subrayar la diferencia entre "probabilidad clásica” y "probabilidad cuántica", escriben Rosenblum y Kuttner:

\begin{abstract}
La probabilidad clásica representa el conocimiento de una situación. Pero esto no es todo. Se presume que existe algo físico además de dicho conocimiento, algo a lo que se le asigna la probabilidad. [...] La probabilidad cuántica, en cambio, es objetiva. Es la misma para todo el mundo. La función de onda es todo lo que hay: la descripción cuántica de la situación física completa no añade un átomo a la función de onda del átomo. (Rosenblum y Kuttner, 2011, p. 96) ${ }^{7}$
\end{abstract}

En Copenhague se describe la forma como Heisenberg elabora el principio de incertidumbre, durante una estadía en Heligoland, una pequeña isla alemana en el mar del Norte. Más exactamente, se trata cómo resuelve el planteamiento que haría posible que "[...] the mathematics first began to chime with the principle" (Frayn, 2000, p. 47). Es la mecánica matricial, de la que aquí no conocemos mayores detalles, salvo que, casi al mismo tiempo, Schrödinger iba a proponer una solución matemática diferente, la mecánica ondulatoria. Y, más importante, se nos hace ver que Heisenberg consideró una afrenta personal el que Bohr, después de discutir agriamente con Schrödinger al respecto (aunque Bohr insiste, no muy verosímilmente, en haber sido siempre muy cortés), ${ }^{8}$ declarara que ambos tenían razón: "I said wave mechanics and matrix mechanics were simply alternative tools" (Frayn, 2000, p. 48). Según Heisenberg, Bohr lo traicionó cuando "pactó" con Schrödinger. Es notable cómo el drama nos hace ver que, tan importantes como los conocimientos mismos de estos físicos, son sus egos: Heisenberg se rehúsa a darle crédito a Schrödinger por razones meramente ligadas a su orgullo personal y espera de Bohr una conducta similar, con total ceguera en relación con los aspectos propiamente matemáticos, menos "importantes" que una supuesta lealtad. Por su parte, Bohr no deja de resentir el que Heisenberg, con quien trabajaba lado a lado cada día, se haya "aprovechado" de una salida a Heligoland coincidente con un viaje de Bohr a Noruega para hacer un primer borrador sobre el principio de incertidumbre y hasta mandarlo a publicar, a sus espaldas: "My dear good Heisenberg, it's not open behavior to rush a first draft into print before we've discussed it together! It's not the way we work! (Frayn, 2000, p. 50) A lo cual, Heisenberg replica: "No, the way we work is that you hound me from first thing in the morning till last thing at night! The way we work is that you drive me mad!" (Frayn, 2000, p. 50). Como Margrethe no se cansa de anotar, ellos no hacían más que enojarse el uno al otro: "I shouldn't let you sit anywhere near each other, if I were the teacher" (Frayn, 2000, p. 50). Así pues, el principio de incertidumbre surge a partir del trabajo conjunto de Heisenberg y Bohr, pero le debe más al primero en su formulación inicial, si bien el segundo es quien encuentra un error en esta que Heisenberg se ve obligado a corregir. Pero lo que más se resalta son los resentimientos que quedan entre ellos a nivel personal: Bohr, quien era el mayor y de algún modo el maestro (se dice que a sus espaldas, los demás físicos del grupo lo llamaban "el papa"), resiente que el discípulo lo haya ignorado a la hora de proponer el principio de incertidumbre; Heisenberg, quien ha pasado por una experiencia de descubrimiento en Heligoland que considera casi mística, resiente que Bohr 
haya pactado con Schrödinger y, quizás más, que Bohr no haya quedado tan extasiado como él ante los alcances de su descubrimiento:

[...] I show him the strangest truth about the universe that any of us has stumbled on since relativity -that you can never know everything about the whereabouts of a particle, or anything else, even Bohr now, as he prowls up and down the room in that maddening way of his [...] I shatter the objective universe around you -and all you can say is that there's an error in the formulation! (Frayn, 2000, p. 51).

Heisenberg atribuye esta miopía de Bohr a los celos, en tanto Bohr está convencido de que el primero fue incapaz de valorar, por la misma razón, la corrección que él efectúa.

Las bases, pues, en las que asentaba la relación, ya desde los años veinte (cuando supuestamente eran colaboradores) se encuentran falseadas. Tienen la forma de una competencia en la que los ataques personales cuentan tanto, o más, que los aportes intelectuales. No en balde recuerdan el modo en que alguna vez los dos compitieron para bajar una montaña esquiando, y cada cual se pretende a sí mismo el ganador: Heisenberg porque fue el más veloz, y Bohr porque hizo el recorrido sabiendo exactamente dónde se encontró en todo momento, sin que mediara la incertidumbre. Incluso antes, el primerísimo encuentro de Heisenberg y Bohr ha estado matizado por la discordia: el joven Heisenberg irrumpió en un homenaje que le rendían a Bohr, para señalarle un error matemático. Y si bien se reconoce que la competitividad feroz (disfrazada tal vez de colaboración feliz, sobre todo por parte de Bohr) da buenos resultados para la ciencia, también queda claro que estos dos hombres no han vivido jamás una época de convivencia pacífica y que su rivalidad siempre ha sido más fuerte que su amistad. ${ }^{9}$

Por su parte, Volpi (2000) aborda la gestación del principio de incertidumbre desde un punto de vista parcialmente distinto. En primer lugar, pone el énfasis en la construcción del ambiente natural de Heligoland en medio del cual Heisenberg recibe la iluminación que le permite crear tal principio. Se elabora un símil en el que las fuerzas de la naturaleza, visualizadas a través de la prosopopeya, sostienen una batalla, que viene a ser una tempestad. Al cabo, se compara esta tempestad con la lucha que Werner Heisenberg ha vivido en el interior de su cabeza. La prosopopeya no deja de incluir elementos que incluso apuntan a una cierta divinización de la naturaleza:

\footnotetext{
La tormenta disminuye poco a poco, reabsorbida por las corrientes náuticas, y el universo comienza su penoso regreso a la calma. Los dos colosos - mar y tierra- recuperan sus posiciones de ataque, dispuestos a concederse una tregua para sanar a los heridos. Como si un dios benevolente quisiese festejar el acuerdo, los nubarrones se abren de pronto [...] (Volpi, 2000, p. 248)
}

Pero no se crea que Volpi se entrega a una lectura mística del entorno natural: está claro que se trata de un símil que no pretende dar cuenta de una cosmología revelada a Heisenberg, sino hacer una elaboración artística que, dando caracteres humanos y hasta divinos a los elementos naturales, quiere tratar de describir lo que estaba ocurriendo en la mente de este científico. El texto insiste en que ahora se centra en la valoración subjetiva que efectúa Heisenberg en relación con el principio de incertidumbre: "Para muchos se tratará sólo de una excusa matemática, de un golpe de genio, de una fórmula más o menos perfectible; sólo él puede estar seguro de que se trata de algo mucho más grande... De la perfección" (Volpi, 2000, p. 250). Sin perder para nada esa apreciación subjetiva de su propuesta científica, no es de extrañar que la actitud que se atribuye en adelante a Heisenberg sea la de quien tiene por delante una misión religiosa: “QQué es un profeta sin alguien capaz de escucharlo? Antes de regresar a Copenhague o a Gotinga o a Múnich, se detendrá en Hamburgo para visitar a Pauli y convertirlo en el primero de sus seguidores, en el profeta de la mecánica matricial [...]" (Volpi, 2000, pp. 250-251). Así pues, 
Heisenberg se retrata como un "iluminado" (si bien se advierte que él mismo jamás habría admitido tal denominación), que ha obtenido una verdad inamovible, perfecta. No resultan al cabo demasiado discernibles, en esta versión de Volpi, el perfil del científico y el del religioso, pero dejando en claro que se trata de que sus vivencias son paralelas (y no sus verdades, valórense estas de la forma en que se valoren).

Otro asunto resulta notable en la versión de Volpi, si la comparamos con la de Frayn: el hecho de que Bohr no aparezca en esta primera parte de la historia, en Heligoland. Es otro físico, Pauli, quien recibe la primera atención de Heisenberg, y no su maestro Bohr. Más adelante, En busca de Klingsor también incluirá la forma en que la mecánica matricial de Heisenberg y la mecánica ondulatoria de Schrödinger entraron en conflicto. La versión se atribuye a este último, a quien se pinta abiertamente como la "némesis" de Heisenberg. Son polos opuestos tanto matemática como existencialmente. Heisenberg trata en su mecánica a la luz en tanto partículas y Schrödinger en tanto ondas,${ }^{10}$ casi como una consecuencia de lo siguiente:

\footnotetext{
[...] Heisenberg era una especie de estoico de la física; Schrödinger representaba su lado hedonista. Sus carreras se habían desarrollado de modo opuesto: mientras Schrödinger había pasado su juventud sin echarle siquiera un vistazo a la nueva teoría cuántica, Heisenberg prácticamente se había formado con ella; del mismo modo, mientras el vienés era sólo un modesto profesor en la Universidad de Zúrich cuando empezó a publicar sus primeros descubrimientos importantes, Heisenberg era una especie de niño prodigio, mimado y protegido por las grandes figuras de la física desde la adolescencia. (Volpi, 2000, p. 262)
}

Según Schrödinger, su mecánica ondulatoria se impondrá sobre la mecánica matricial de Heisenberg fundamentalmente por su mayor simpleza matemática, y esto pese a las influencias de Heisenberg (a quien llama "[...] mimado por todos los grandes como Bohr, Sommerfeld y Born" (Volpi, 2000, p. 272)), mucho mayores que las suyas. De todos modos, Schrödinger explica que él mismo demostró que ambas teorías eran equivalentes en un artículo de 1926, pero que la suya era mucho más aplicable que la de Heisenberg, matemáticamente hablando, por lo que, aunque este no dejó de manifestar su ira por todos los medios posibles (incluso increpándolo personalmente), el tiempo terminó por darle la razón. Está claro, por lo demás, que en esta versión de Volpi (según la óptica del personaje de Schrödinger, por supuesto), Bohr tomó el partido de Heisenberg, su protegido, y se encargó de acosar a Schrödinger en una guerra que le declaró abiertamente. ${ }^{11}$

También incluye Volpi la versión del propio Bohr sobre la creación del principio de incertidumbre, y aunque se admite que tuvo algunas diferencias con Heisenberg a raíz del artículo pergeñado en Heligoland, aparecen bastante suavizados los problemas o las rivalidades entre ellos durante los años veinte. Bacon le plantea que él y Heisenberg colaboraban y luchaban, a lo que Bohr replica que más bien jugaban, y que la ciencia surge del caos y del conflicto, y no de la tranquilidad o la paz. Aunque hubiese "errores técnicos" en el artículo detectados entonces por Bohr, para molestia de Heisenberg, se asume que ellos muy rápidamente superaron los conflictos personales que pudieron haberse creado durante esa década. Una versión, pues, bastante distinta de la que nos da Frayn.

Me refiero ahora al principio de complementariedad, la creación de Bohr. Nos explican Rosenblum y Kuttner, dos físicos que insisten en el carácter misterioso de la mecánica cuántica, las motivaciones de Niels Bohr para crearlo:

[...] comprendió que, para que los físicos se permitieran continuar haciendo física sin empantanarse en la filosofía, tenía que afrontar la influencia del conocimiento en los fenómenos físicos. Fruto de esta inquietud fue su principio de complementariedad: los dos aspectos de un objeto microscópico, el de partícula y el de onda, son 'complementarios', y una descripción completa requiere ambos aspectos contradictorios, pero debemos considerar sólo un aspecto a la vez. (Rosenblum y Kuttner, 2011, p. 134) 
Se trató, para Bohr, de que había que tomar en cuenta que el sistema microscópico no existe por sí solo, sin la presencia perturbadora del observador, el cual es un objeto macroscópico: "Siempre debemos incluir en nuestra discusión, al menos implícitamente, los diferentes aparatos macroscópicos empleados para evidenciar cada uno de los aspectos complementarios." (Rosenblum y Kuttner, 2011, p. 134) Estos aparatos macroscópicos exhiben un comportamiento "clásico" (en el sentido más o menos opuesto a comportamiento "cuántico") y sólo pueden dar cuenta del objeto microscópico en tanto onda o en tanto partícula, ya que así es como funcionan los objetos macroscópicos. Esto parece tener una implicación más o menos grave en el pensamiento de Bohr, pues, de acuerdo con estos autores:

\begin{abstract}
...aunque los físicos hablan de los átomos y otras entidades microscópicas como si fueran cosas físicamente reales, Bohr nos dice que las cosas microscópicas son sólo conceptos que empleamos para describir el comportamiento de nuestros instrumentos de medida. No son objetos con una realidad independiente, como los guisantes o las piedras, de los que podemos hablar directamente. (Rosenblum y Kuttner, 2011, pp. 134-135)
\end{abstract}

Este, si se lo quiere llamar así, "extremo" del pensamiento de Bohr, es lo que parece haber creado, al menos inicialmente, un resquemor en Heisenberg:

\begin{abstract}
Un colega de Heisenberg sugirió una vez que el problema de la dualidad onda-partícula era una cuestión puramente semántica que podría resolverse diciendo que los electrones no eran ondas ni partículas, sino "ondículas". Heisenberg, insistiendo en que las cuestiones filosóficas planteadas por la mecánica cuántica incluían lo grande además de lo pequeño, replicó: No, esa solución es un poco demasiado simple para mí. Después de todo, no estamos tratando con una propiedad especial de los electrones, sino con una propiedad de toda la materia y toda la radiación. Tomemos electrones, cuantos de luz, moléculas de benzol o piedras, siempre iremos a parar a estas dos características, lo corpuscular y lo ondulatorio. (Rosenblum y Kuttner, 2011, p. 130).
\end{abstract}

Como se ve, al menos sobre este punto, pareciera que hubo un desacuerdo teórico entre Bohr y Heisenberg.

De tal cosa da cuenta la versión de Michael Frayn. Aquí, Bohr insiste en la complementariedad: "[...] to understand how people see you, ${ }^{12}$ we have to treat you not just as a particle, but as a wave" (Frayn, 2000, p. 52) Y, aunque Heisenberg asiente, y afirma haberlo añadido en la postdata de su artículo, Bohr mantiene que, en primer lugar, todos recuerdan el artículo (el mismo que Bohr le reclama haber publicado en su ausencia), pero nadie recuerda la postdata, y, en segundo lugar, Heisenberg no aceptó jamás, por entero, la complementariedad, que es al fin y al cabo lo que Bohr comenta aquí y lo que fue su contribución fundamental. Heisenberg niega no estar convencido, pero enseguida sale a colación el hecho de que fue necesaria la mediación de Pauli:

\footnotetext{
Heisenberg: We had to drag Pauli out of bed in Hamburg once again to come to Copenhagen and negotiate peace. / Bohr: He succeeded. We ended up with a treaty. Uncertainty and complementarity became the two central tenets of the Copenhagen Interpretation of Quantum Mechanics. / Heisenberg: A political compromise, of course, like most treaties. / Bohr: You see? Somewhere inside you there are still secret reservations. (Frayn , 2000, p. 53)
}

Y así, permanece la sombra de que Heisenberg mantiene su desacuerdo, al menos íntimamente. Llama la atención el hecho de que la posición científica de ambos personajes es notoriamente influida por su necesidad de acuerdos "políticos", en donde median consideraciones personales e intereses particulares, lo cual reafirma Margrethe, al comentar que Heisenberg obtiene su cátedra en Leipzig por cuanto la doctrina de Copenhague se establece sólidamente gracias a estos acuerdos. 
Por su parte, En busca de Klingsor pone en boca de Bohr lo que fue la creación del principio de complementariedad y, como en la versión de este texto de la creación del principio de incertidumbre que ya comentamos, pasa por alto los posibles desacuerdos entre Bohr y Heisenberg. Más bien, insiste en que el oponente principal del desarrollo que siguió la mecánica cuántica era Einstein, mientras que Bohr y Heisenberg se mantenían en un frente unido:

[...] nuestras ideas sobre la mecánica cuántica terminaron por imponerse sobre las teorías rivales. /
- ¿“Nuestras ideas", profesor -se atrevió a preguntar Bacon./ Bohr dudó un instante./ - Las de Heisenberg,
las de Pauli y las mías -respondió al fin-. Eso era el espíritu de Copenhague... Nosotros tres contra el
mundo, querido amigo. Sólo nosotros. (Volpi, 2000, p. 314)

Volpi reconstruirá, pues, el encuentro de 1941 sobre una base muy distinta a la de Frayn: más bien plantea que, junto a Pauli, Heisenberg y Bohr habían logrado un entendimiento tanto científico como existencial sumamente sólido durante los años veinte. Además, la versión que ofrece Volpi sobre el principio de complementariedad también tiene una diferencia con respecto a la de Frayn, desde el punto de vista científico: mientras este llama "complementariedad" al principio según el cual ha de considerarse a un objeto microscópico como onda y como partícula, Volpi llama "complementariedad" al principio según el cual en las consideraciones del científico este debe tomar en cuenta que su propia interacción con el mundo cuántico se efectúa por medio de mediciones clásicas (vale decir, mediciones dadas por objetos macroscópicos), de modo que la física cuántica y la clásica, en la práctica, se complementan:

\footnotetext{
- ¿Pero no sería posible considerar los aparatos de medición, un telescopio, por ejemplo, como objetos que también están sometidos a la mecánica cuántica? / -Sí, sería posible -admitió Bohr-. Pero entonces habría que olvidarse de las descripciones limitadas que nos proporciona de acuerdo con la mecánica clásica. El problema aparece cuando tenemos que registrar las propiedades mecánicas del telescopio. Para hacerlo, necesitaríamos otro aparato en el cual volverían a aparecer mediciones clásicas. ¿Se da cuenta? La serie se vuelve interminable a menos que en algún momento estemos dispuestos a aceptar una medida clásica... La idea de observación se vuelve entonces tan arbitraria que depende de cuántos observadores se consideren incluidos en el sistema observado... [...] No hay más solución que aceptar que la física clásica y la física cuántica se complementan. (Volpi, 2000, pp. 312-313)
}

La cita es extensa, pero se justifica porque demuestra un punto medular para nuestra investigación: que las diferencias entre la exposiciones de la práctica científica ofrecidas en los textos literarios no implican tan sólo aquellos aspectos que alguien podría calificar de subjetivos (por ejemplo, hasta ahora, la clase de relaciones personales establecidas entre Bohr y Heisenberg durante los años veinte: ¿había más celos que colaboración, más rivalidad que amistad?), sino también aquellos que finalmente tampoco son objetivos (aunque podría creerse que sí): Frayn expone el principio de complementariedad de una forma y Volpi de otra, al menos en cuanto a los énfasis con que debe interpretársele. ${ }^{13} \mathrm{Y}$ si bien, hasta donde me es posible visualizar, existe más bibliografía científica que se inclina por la versión de Frayn (complementariedad de la onda y la partícula, y no complementariedad de la física clásica y la cuántica), no deja de existir también un margen interpretativo, pues muchos concuerdan en que el pensamiento de Bohr no es nada transparente, como hubiera podido esperarse si se tratase de una muestra de eso tan imprecisamente llamado "conocimiento objetivo": "El pensamiento de Bohr es notoriamente difícil de aprehender", comentan Rosenblum y Kuttner (2011, p. 130), precisamente al preguntarse si Niels Bohr llegó a negar que la ciencia pudiera describir cómo efectivamente es la naturaleza, y en su lugar propuso que la física sólo tiene que ver con lo que podemos decir de la naturaleza. ${ }^{14}$ 
El episodio de En busca de Klingsor en que aparece Bohr incluye una comparación sumamente interesante entre el texto científico de la mecánica cuántica (en particular, su búsqueda del electrón) y el texto policiaco de la narrativa literaria (en particular, el propio texto de la novela que estamos leyendo). Figuradamente, el electrón es Klingsor, ese criminal escurridizo que no se deja atrapar porque su comportamiento es notoriamente desconcertante en relación con lo que estábamos acostumbrados a esperar de los criminales tradicionales. Si -como efectivamente sucederá- no podremos saber quién es Klingsor sin un margen de incertidumbre que deja a esta novela policiaca (aunque esta clasificación la describe tan sólo parcialmente) con un final abierto que quizás no complazca a los fanáticos del subgénero, ello puede derivarse del paralelismo aquí planteado entre Klingsor y el electrón: este, a partir de la mecánica cuántica, ha sido visualizado como un ente que (a diferencia de los "bandidos menores" con los que se enfrentó Newton) "[...] no sólo huye y desaparece, sino que al hacerlo infringe todas las leyes conocidas" (Volpi, 2000, p. 315). La mecánica cuántica se constituye como un esfuerzo por localizar e identificar al electrón y apenas ofrece resultados incipientes: "[...] nos revela su posición sin decirnos adónde se dirige, o viceversa...” (Volpi, 2000, p. 315) (Recordemos que este tipo de afirmación corresponde al principio de incertidumbre de Heisenberg.)

Esta última comparación entre el electrón y un elusivo criminal nos remite a la versión que Volpi da del que, según Rosenblum y Kuttner, viene a ser el tercer pilar de la interpretación de Copenhague: la interpretación probabilística de la función de onda ${ }^{15}$ : "La ondulatoriedad en una región es la probabilidad de encontrar el objeto en esa región" (Rosenblum y Kuttner, 2011, p. 94). "Esta interpretación probabilística de la ondulatoriedad ocupa un lugar central enla interpretación de Copenhague. Mientras que la física clásica es estrictamente determinista, la mecánica cuántica nos habla de la aleatoriedad última de la Naturaleza.” (Rosenblum y Kuttner, 2011, p. 126) Volpi lo ha planteado así: “[...] la mecánica cuántica prefiere determinar, estadísticamente, cuáles son las guaridas más probables en que el electrón decidirá esconderse una vez consumadas sus fechorías" (Volpi, 2000, p. 315).

Frayn, por su lado, no aborda sino tangencialmente este aspecto estadístico de la mecánica cuántica, pues parece centrarse más en lo que Heisenberg y Bohr consiguieron por separado (incertidumbre y complementariedad), que en esta versión creaba distanciamiento personal entre ellos. El texto de Frayn ha optado, como ya se ha visto, por subrayar la rivalidad entre estos personajes, y por ello también escoge cuál énfasis da a la lectura del conocimiento científico. La interpretación probabilística de la función de onda, en tanto no fue objeto de disputa entre Heisenberg y Bohr, tampoco es objeto de interés para el drama de Frayn.

\section{Los principios antrópicos}

En términos muy generales, el principio antrópico es "...la idea de que podemos alcanzar conclusiones sobre las leyes aparentes de la física a partir del hecho de que existimos" (Hawkins y Mlodinow, 2010, p. 210). El planteamiento, que en un inicio puede parecer incluso perogrullesco (¿cómo conseguiríamos pensar nada acerca de la física o de cualquier otra materia si no existiéramos?) tiene numerosas implicaciones complejas. El llamado principio antrópico "débil" se refiere, según Hawkins y Mlodinow (2010, p. 174), a "[...] cómo nuestro conocimiento de nuestra propia existencia impone reglas que seleccionan, de todos los entornos posibles, sólo aquellos que permiten la vida." Por ello estos dos científicos proponen que el principio debería llamarse "de selección": al fin y al cabo, sirve para entender por qué, 
entre muchas posibles, se han seleccionado ciertas regulaciones universales que han dado lugar a un entorno "amigable" con nuestra propia existencia: de no existir estas regulaciones, tampoco estaríamos haciéndonos la pregunta de por qué se dan, ya que no podríamos hacernos pregunta alguna. Es una propuesta que conlleva implicaciones tanto abstractas y metafísicas (¿acaso existe un "seleccionador" inteligente, es decir, Dios?) como prácticas y concretas: por ejemplo, estos mismos investigadores nos explican la forma en que el principio antrópico débil puede utilizarse para calcular la edad del universo a partir del hecho de que nuestra existencia solamente se puede producir por la presencia de elementos como el carbono, el cual requiere de una cierta "cocción" lenta en las estrellas, una diseminación y una condensación en un espacio planetario, todo lo cual necesita, según los cálculos actuales, de unos diez mil millones de años. "Por otro lado, el universo no puede ser mucho más viejo que diez mil millones de años, ya que en el futuro lejano se habrá consumido el combustible para las estrellas, y necesitamos estrellas calientes para nuestro sostenimiento" (Hawkins y Mlodinow, 2010, p. 175).

Se considera el principio antrópico como "débil" en la medida en que se supone que las restricciones o selecciones impuestas tienen un ámbito de acción prioritario en nuestro entorno. Ahora bien, cuando se considera que las restricciones afectan a todas las leyes de la naturaleza, se le llama principio antrópico "fuerte":

Esta idea surgió porque no son sólo las peculiares características de nuestro sistema solar las que parecen extrañamente compatibles con el desarrollo de la vida humana, sino también las características del conjunto del universo, y eso es mucho más difícil de explicar. (Hawkins y Mlodinow, 2010, p. 176)

No existe un acuerdo entre los mismos físicos actuales acerca de cuánta importancia debe concedérsele al principio antrópico, en especial el fuerte. Muestras de hasta dónde puede llegar este desacuerdo las proporcionan las siguientes citas. La primera procede de Martin Rees, catedrático de la Universidad de Cambridge y astrónomo real de Inglaterra:

En el principio sólo había probabilidades. El universo sólo podía acceder a la existencia si alguien lo observaba. No importa que los observadores aparecieran varios miles de millones de años más tarde. El universo existe porque tenemos conciencia de él. (en Rosenblum y Kuttner, 2011, p. 239) ${ }^{16}$

La segunda, de Murray Gell-Mann, premio Nobel de física y descubridor del quark: "[...] en cualquier caso, al universo le trae sin cuidado que unos seres humanos surgidos en un oscuro planeta estudien su historia, y seguirá obedeciendo las leyes mecanocuánticas de la física independientemente de la observación a que se le someta." (Gell-Mann, 2007, p. 156) También pueden citarse a quienes, como John Wheeler, defienden una "visión participativa" de la realidad:

El sistema de experiencia compartida que llamamos mundo se contempla como una autoconstrucción de fenómenos cuánticos elementales, actos elementales de participación observacional. En otras palabras, las preguntas que los participantes plantean (y las respuestas que obtienen) mediante sus dispositivos observacionales, más la comunicación de sus hallazgos, intervienen en la creación de las impresiones que llamamos el sistema: ese gran sistema global que a primera vista es tiempo y espacio, partículas y campos. (en Rees, 2001, p. 263) ${ }^{17}$

Así pues, nos hallamos en un territorio propicio para la polémica. No en balde, ha dado lugar, entre otros planteamientos, a uno que hemos seguido bastante a la hora de redactar este artículo, el de Rosenblum y Kuttner en su libro El enigma cuántico. Estos autores nos hacen ver cómo las implicaciones de la importancia del observador en el mundo cuántico son de carácter filosófico, incluso metafísico: 
La existencia de un enigma no es una cuestión física. Es metafísica en el sentido original de la palabra. [...] Cuando se trata de metafísica, los no físicos con una comprensión general de los hechos experimentales -sobre los que no hay discusión- pueden tener una opinión tan válida como los físicos. (Rosenblum y Kuttner, 2011, p. 110)

Todos quedamos llamados a opinar acerca de un problema ciertamente misterioso, pues no se trata de simplemente aceptar la importancia de la visión subjetiva del observador de los sucesos físicos, en particular los cuánticos. Si así fuera, no se estaría planteando nada nuevo ni enigmático. Se trata de que la realidad cuántica afectada por la observación es una realidad objetiva: "Una observación crea una situación objetiva, que es la misma para todo el mundo" (Rosenblum y Kuttner, 2011, p. 122). Rosenblum y Kuttner nos explican la demostración experimental de tal aseveración, en el mundo de lo microscópico, ${ }^{18}$ y además nos hacen ver cómo la experimentación actual va tras la ruptura de la frontera entre lo macroscópico y lo microscópico, o al menos hacia su dinamización:

[...] los fenómenos cuánticos [...] están bien establecidos para objetos muy pequeños. Estos fenómenos se están evidenciando en objetos cada vez más grandes. En la actualidad, se está experimentando con proteínas de tamaño medio. ¿Serán los virus los próximos? La teoría cuántica no impone límites.

El tamaño de los objetos susceptibles de evidenciar efectos cuánticos parece restringido sólo por la tecnología y el presupuesto. (Rosenblum y Kuttner, 2011, p. 110)

En esta dirección, pareciera que la "complementariedad", tal como la expone Volpi, es decir, la necesidad de que la experimentación ante lo microscópico y cuántico se complemente con la experimentación ante lo macroscópico y clásico, va camino de debilitarse. Pero bueno, recordemos que esa es solamente la lectura que del principio de complementariedad de Bohr realiza Volpi, y que la mayoría de los autores consultados hablan de este concepto en términos de complementariedad entre mecánica de partículas y mecánica ondulatoria (Rosenblum y Kuttner [2011], Hawkins y Mlodinow [2010], Gell-Mann [2007]).

La importancia concedida al rol del observador resalta en la novela En busca de Klingsor desde el primer momento. Es un texto construido desde la plena conciencia del "principio antrópico" del arte: la realidad existe porque el observador interviene en ella para crearla. Sólo podemos conocer una realidad novelesca porque esta existe como parte de la experiencia humana de la producción verbal. Sí, la realidad es texto en una novela y esto parecería ser una observación perogrullesca: todo texto se escribe por parte de alguien en concreto, que no puede sino "antropizar" lo que dice. Pero recordemos que, como planteamiento inicial, el principio antrópico también resultaba un tanto perogrullesco. Otra cosa se da cuando se reflexiona un tanto más. Y esto es exactamente lo que hace Volpi, en particular al inicio del Libro Primero, cuando expone las "Leyes del movimiento narrativo".

Son tres: "Toda narración ha sido escrita por un narrador", "Todo narrador ofrece una verdad única" y "Todo narrador tiene un motivo para narrar". Volpi es el primero en señalar ${ }^{19}$ que estas leyes parecen verdades de Perogrullo... exactamente igual a como ocurre con el principio antrópico en física: "Esta aseveración, que a primera vista parece no sólo tautológica sino decididamente estúpida, es más profunda de lo que se suele admitir." (Volpi, 2000, p. 23). La profundidad proviene del hecho - bien establecido en el Corolario I de esta misma secciónde que el autor, de carne y hueso, cede la palabra a un narrador, ente ficcional, que se pretende autor del escrito dentro del mundo narrativo. Nos hallamos ante una disyuntiva no demasiado diferente de la que exponíamos al hablar de "principio antrópico débil" y "principio antrópico fuerte". Si aceptamos solamente el principio antrópico débil, diremos que el mundo narrativo se visualiza y se presenta desde la perspectiva de un narrador ficcional, pero si vamos más 
allá, al fuerte, diremos que en realidad el lenguaje literario sólo puede existir como resultado de la intervención de un autor. ¿Puede el autor ceder verdaderamente la palabra a un narrador ficcional, lo que haría que ciertas "leyes" particulares rigieran únicamente en el entorno particular de lo que este narrador alcanza? ${ }^{20}$ ¿O hay que extender los alcances de la particular posición del narrador ficcional a la más general posición del autor y pensar que todo lo que proviene de la pluma de Volpi implica por igual la imposición de sus "leyes" de autor? ${ }^{21}$ El tema queda abierto, ciertamente. Al final, Volpi se permite una intervención directa, en tanto autor, lo cual parece inclinar la balanza hacia el principio antrópico débil, pero no de una manera tajante: "A pesar de la opinión contraria de Gustav Links, este libro es, fundamentalmente, una novela" (Volpi, 2000, p. 441: es una "Nota final" que firma "J.V.", lo cual claramente establece la ficcionalidad -al menos fundamental, ya que no total, nueva ambigüedad que permite no cerrar el tema- del mundo narrado.)

La segunda de las leyes del movimiento narrativo se relaciona directamente con la importancia del observador en la mecánica cuántica. Aunque se admiten antecedentes desde la Grecia clásica y se resalta aquí el perspectivismo literario de Henry James, lo cierto es que el crédito primordial se le otorga a Erwin Schrödinger:

Cada observador -no importa si contempla un electrón en movimiento o un universo entero- completa lo que Schrödinger llamó el "paquete de ondas" que proviene del ente observado. Al interactuar sujeto y objeto se produce una mezcolanza indefinible entre ambos... (Volpi, 2000, pp. 24-25)

Ahora bien, la visión de Volpi de la importancia de la observación en la mecánica cuántica es menos tajante de lo que la propia mecánica cuántica permite plantear, según hemos visto. Pues Volpi insiste en que Schrödinger "relativiza" la verdad: cada cabeza es un mundo, nadie tiene una verdad mejor que otra, la verdad es $m i$ verdad, y punto.., Pero lo cierto es que la mecánica cuántica es sumamente enigmática por cuanto pretende que la observación crea la realidad objetivamente. Quizás habría incluso que superar esta forma de expresarse pues, de algún modo, pareciera que dentro del modo de pensar propuesto por la mecánica cuántica, la diferenciación entre lo subjetivo y lo objetivo pierde validez, lo cual va mucho más allá de los lugares comunes subjetivistas a que aluden la Ley II, ya citada, y su respectivo Corolario II. ${ }^{22}$ Tengo que decir entonces que, en mi opinión, Volpi se queda corto en su explicación de Schrödinger pues no ve lo que hay de más "escandaloso" en la participación del observador según la mecánica cuántica, y es justamente el hecho de que no se trata más de admitirle a cada cual su derecho de ver las cosas desde el ámbito de su subjetividad (esta sí es una especie tan vieja que ya andaba en la Grecia clásica y, por supuesto, en Henry James), se trata de que el observador asume un rol que sólo la mecánica cuántica le ha conferido. Ahora bien, he de admitir que Volpi tiene todo el derecho de inclinarse por versiones menos polémicas en relación con este tema, como, por ejemplo, la que más arriba comenté, al citar a Murray Gell-Mann (2007). ${ }^{23}$

En Copenhague también se señala la importancia del observador. Desde muy temprano en la obra, Margrethe hace ver que la conversación que Bohr y Heisenberg tuvieron cuando salieron a caminar, sin que ella estuviera presente, tuvo que ser distinta de cualquier otra conversación que ella presenciara, y las razones para ello provienen directamente de la mecánica cuántica: "So they're walking again. He's done. And if they're walking they're talking. Talking in a rather different way, no doubt $-\mathrm{I}^{\prime}$ ve typed out so much in my time about how different particles behave when they're unobserved..." (Frayn, 2000, p. 25). Esta conversación es medular: al fin y al cabo, todo el enigma o gran parte de él, en esta obra, se vería aclarado si pudiéramos presenciar directamente, sin intervenir, la conversación entre Bohr 
y Heisenberg fuera de la casa, cuando salen a caminar, en 1941. Pero la obra elude el presentar tal, precisamente porque es una ilusión pensar que alguien puede "observar directamente, sin intervenir" la realidad: el observador crea la realidad. Esta sería la razón de fondo de por qué es tan difícil (por qué es, en rigor, imposible) saber qué pasó durante esa conversación. Los observadores crean la realidad y los involucrados no la pueden observar, por razones que Bohr, autor de la complementariedad, debería saber. Es Margrethe de nuevo quien declara:

\footnotetext{
If it's Heisenberg at the centre of the universe, then the one bit of the universe that he can't see is Heisenberg. [...] So it's no good asking him why he came to Copenhagen in 1941. He doesn't know. [...] I've typed out often enough. If you're doing something you have to concentrate on you can't also be thinking about doing it, and we did. (Frayn, 2000, pp. 53-54)
}

La complementariedad también se invoca para explicar la gran variedad de lecturas que el episodio puede llegar a proyectar sobre la personalidad y las actuaciones de los participantes. Dice Heisenberg: "Complementary, once again. I'm your enemy; I'm also your friend. I'm a danger to mankind; I'm also your guest. I'm a particle; I'm also a wave” (Frayn, 2000, p. 57).

Pero lo que realmente resalta en Copenhague es la importancia que concede al principio antrópico. El ser humano retoma su centralidad en la concepción del universo a partir de los descubrimientos de la física moderna, según la lectura que, en este texto, hacen Bohr y Heisenberg. Dice el primero: "Not to exagerate, but we turned the world inside out! Yes, listen, now it comes, now it comes... We put man back at the centre of the universe. Througout history we keep finding ourselves displaced" (Frayn, 2000, p. 53). Bohr repasa enseguida cómo la historia de la humanidad ha pasado sólo por breves periodos, como la Grecia antigua y el Renacimiento, en los que se ha creído firmemente en la posición central del ser humano, medida de todas las cosas. La mayoría del tiempo, en los periodos teocéntricos como la Edad Media y en la época posterior al Renacimiento y hasta el siglo XX, el hombre ha vivido postrado ante las evidencias de su propia pequeñez. Entonces el panorama varía, comenzando con Einstein: He shows that measurement -measurement, on which the whole possibility of science depends-
measurement is not an impersonal event that occurs with impartial universitality. It's a human act, carried
out from a specific point of view in time and space, from one particular viewpoint of a possible observer.
(Frayn, 2000, p. 53).

Y luego viene la intervención decisiva de ellos, los físicos de Copenhague:

Then, here in Copenhagen in those three years in the mid-twenties we discover that there is no precisely determinable objective universe. That the universe exists only as a series of approximations. Only within the limits determined by our relationship with it. Only through the understanding lodged inside the human head. (Frayn, 2000, p. 53)

Finalmente, Copenhague se cierra con una serie de consideraciones por parte de los personajes que tienen como base el principio antrópico en su versión más fuerte: el universo como una creación dependiente de la existencia de observadores inteligentes. No se lo plantea como una certeza, sino más bien como una posibilidad. Dice Margrethe, reflexionando sobre las consecuencias de un holocausto nuclear en el cual, al desaparecer la humanidad, desapareciera también el universo entero: "And if we really are the centre of the universe, if we really are all that's keeping it in being, what will be left?" (Frayn, 2000, p. 58). Su marido le responde: "Darkness. Total and final darkness" (Frayn, 2000, p. 58). Toda la obra cierra sobre la melancólica reflexión de que la nada en que la humanidad se ha se sumir (más tarde o más temprano, haya bombas nucleares estallando o no) será la única capaz de acabar con la incertidumbre, porque acabará también con el conocimiento. 


\section{La interpretación de Copenhague}

Para la historia política, y en particular para la historia de la Segunda Guerra Mundial, los acontecimientos de Copenhague en 1941 (vale decir, fundamentalmente el encuentro entre Heisenberg y Bohr) son sumamente relevantes, tanto que entonces pudo gestarse el porvenir de la investigación de los físicos que dio lugar a la bomba atómica de 1945. (Así sería, más probablemente, del lado alemán: lo cierto es que Bohr no estaba a la cabeza de lo que ocurría en Los Álamos, mientras que Heisenberg sí dirigía ciertas operaciones que hubieran podido derivar en una bomba atómica alemana). No hay ninguna certeza de ello, sin embargo: nos movemos en un terreno en el que sólo existen las hipótesis. Pero no es esto lo que ha solido llamarse "interpretación de Copenhague", sino más bien la interpretación que, bajo la dirección de Bohr, se dieron los físicos de un hecho desconcertante, como es el que la observación crea la realidad observada. Esta interpretación data de los años veinte; Rosenblum y Kuttner no dudan en llamarla la "postura ortodoxa de nuestra disciplina” (2011, p. 123).

Lo primero que debe subrayarse es que, contrario a lo que ocurría con la mecánica clásica o newtoniana, e incluso con la relatividad einsteiniana, estamos ante una formulación teórica y una serie de comprobaciones empíricas de tal que necesitan ser interpretadas. La razón de esta necesidad se encuentra en la extrañeza que la evidencia cuántica produce, la cual debe encontrar un asidero filosófico (o, si se quiere, metafísico) nada evidente. El hecho mismo de hablar de una "interpretación" ya le parecerá problemático a quien piense que una ciencia "dura" como la física no debería ser objeto de lecturas (pues una interpretación nunca puede ser única; por definición, las lecturas son plurales); ilusión esta que en tiempos de Newton resistía o se hacía resistir con bastante suerte.

Rosenblum y Kuttner tampoco dudan en afirmar que la interpretación de Bohr y asociados "[...] rebaja [cursiva agregada] la afirmación de que la observación produce las propiedades observadas estableciendo que una observación tiene lugar allí donde un objeto microscópico a escala atómica interacciona con un objeto macroscópico.” (2011, p. 124).

El corolario es:

Así pues, la interpretación de Copenhague considera dos dominios: el dominio macroscópico clásico
de nuestros instrumentos de medida, regido por las leyes de Newton, y el dominio microscópico
cuántico de los átomos y otros objetos muy pequeños, regido por la ecuación de Schrödinger. Según
esta interpretación, nunca tratamos directamente con los objetos cuánticos del dominio microscópico,
así que no tenemos que preocuparnos por su realidad -o irrealidad- física. Todo lo que necesitamos
es una "existencia" que permita el cálculo de sus efectos sobre nuestros instrumentos macroscópicos.
(Rosenblum y Kuttner, 2011, p. 124)

Queda claro, entonces, que lo que esta lectura de Bohr y asociados hace es, en buena parte, eludir el desconcierto filosófico que el rol de la observación produce, limitándose y limitándonos a creer que no hace falta pensar sobre el estatuto ontológico de los objetos cuánticos, por la sencilla razón de que jamás tratamos de forma directa con estos objetos, sino sólo mediante los objetos macroscópicos sobre los cuales los objetos cuánticos producen efectos. Esta posición es ciertamente pragmática: se impone una frontera formulada a partir del límite de lo que la experimentación puede arrojar como resultado. No sólo Rosenblum y Kuttner expresan reservas entre los expertos que he consultado; sobre este punto, incluso Murray Gell-Mann dice:

Esta interpretación original de la mecánica cuántica, restringida a la repetición de experimentos realizados por observadores externos, es demasiado estrecha para resultar aceptable hoy día como caracterización fundamental, especialmente desde que se ha ido aclarando que la mecánica cuántica debe aplicarse al universo como un todo. (Gell-Mann, 2007, p. 155) 
Parece entonces que Bohr y asociados tomaron la decisión de dejarse guiar por un cierto pragmatismo antes que "empantanarse" en una discusión filosófica que no iban a poder resolver. Esto se resalta ciertamente en Copenhague, donde curiosamente es Heisenberg, que no Bohr, quien aparece como el espíritu amante del pragmatismo más radical; sus razones para aceptar la interpretación se reducen a una palabra: esta funciona. Lo que conlleva la mecánica cuántica como trasfondo filosófico no tiene para Heisenberg la importancia que sí tiene para Bohr, quien, aunque admite que si la interpretación de Copenhague se aceptó fue por razones pragmáticas (“...it was the only way to explain what the experimenters had observed." (Frayn, 2000, p. 55)), se nos aparece como un espíritu mucho más sensible a la especulación, incluso cuando esta apertura de pensamiento conlleva el enfrentarse con la contradicción:

\footnotetext{
Heisenberg: You actually loved the paradoxes, that's your problem. You revelled in the contradictions.

/ Bohr: Yes, and you've never been able to understand the suggestiveness of paradox and contradiction.

That's your problem. (Frayn, 2000, p. 49)
}

Por su parte, y como ya lo he adelantado, Volpi aborda con el nombre de "complementariedad" justamente esta "interpretación de Copenhague", sobre la cual el autor mexicano agrega datos circunstanciales como su presentación por parte de Bohr en congresos en Como y Solvay, en 1927 (Volpi, 2000, pp. 312 ss). En la versión de En busca de Klingsor, el gran opositor de esta interpretación, que no provoca roce alguno ni siquiera en cuanto a matices o detalles entre Bohr y Heisenberg, es Einstein. Se trata de que Einstein se oponía en rigor a toda la mecánica cuántica, y la opinión de Bohr es que lo hacía por haberse quedado atrás: "Él seguía demasiado obsesionado con la claridad de la física clásica para aceptar los desafíos conceptuales de la nueva era" (Volpi, 2000, p. 313). Aunque Bohr mismo declara no entender el punto de vista alternativo que Einstein quería comprobar en relación con la mecánica cuántica (ni siquiera está seguro de que Einstein tuviera alguno), lo que sí se le trasluce es que Einstein estaba molesto por el papel que el azar jugaría dentro de la realidad según esta disciplina, y además, Einstein no iba a poder conformarse con la interpretación de Copenhague, que en aras del pragmatismo sacrificaba la reflexión filosófica más de fondo: "La mecánica cuántica es muy impresionante [...], pero una voz interna me indica que aún no se trata de la verdad. La teoría funciona, pero difícilmente nos acerca al secreto del Viejo. Estoy convencido de que Él no juega a los dados" (Volpi, 2000, p. 313: se trata aquí de Bohr citando una carta de Einstein a Max Born). Einstein, proclive siempre a la reflexión filosófica incluso de ribetes teológicos, no puede admitir la postura pragmática de dar por bueno lo que, sencillamente, funciona, pero no se explica cómo funciona.

\section{El segundo encuentro propiamente dicho: Copenhague, 1941}

En lo que respecta al encuentro concreto de Bohr y Heisenberg en 1941, todo lo anterior no debe verse como mero escenario preparatorio de este, sino más bien como un trasfondo filosófico y científico de la mayor importancia. Sin embargo, nos resta aún comparar las versiones de Frayn y Volpi en relación con los hechos ocurridos en la visita de Heisenberg a Bohr, durante la ocupación alemana de Dinamarca.

La estrategia de Copenhague es, como ya hemos visto, no presentar jamás directamente el encuentro, al menos en lo que respecta a la conversación de Heisenberg y Bohr a solas, cuando caminaban fuera de la casa de este, posiblemente persuadidos de que adentro habría micrófonos nazis escuchándolos. Con ello, en primer lugar, se subraya la imposibilidad de una observación "no 
interventora", como ya he comentado. Por otro lado, se elaboran, por parte de ambos personajes (y, en segundo término, por parte de Margrethe) una serie de versiones sucesivas acerca de lo que ocurrió en ese encuentro, al modo de cómo se construye un artículo científico. ${ }^{24}$ Pero lo que mejor se hace ver en Copenhague es la imposibilidad de superar un margen de incertidumbre en relación con lo ocurrido. En este sentido, se trata de una pieza performativa, pues no sólo habla acerca de la incertidumbre, sino que la pone en escena. Lo hace tomando en cuenta la especial noción de incertidumbre propia de la mecánica cuántica, y no un sentido cualquiera del término: de haber sido este el caso, bastaría con crear una serie de dudas y relativizar las posibles explicaciones, dando a cada cual un cierto crédito. Pero Frayn quiere poner en escena la incertidumbre de Heisenberg: aquella según la cual es posible conocer una variante a la vez, ignorando las demás. Así, cuando conocemos lo que Heisenberg dijo a Bohr (la pregunta de si los físicos tenían el derecho moral de trabajar en la explotación de la energía atómica), ignoramos las implicaciones de la pregunta: ¿se refiere Heisenberg a construir un reactor nuclear o ve posible la creación una bomba atómica? Incertidumbre. Cuando conocemos que Heisenberg no deseaba colaborar con Hitler en la creación de armas nucleares, ignoramos si con su visita a Bohr pretendió que este tampoco colaborase con los aliados en el afán de estos por construir la bomba atómica. Y cuando conocemos que Heisenberg no hizo adecuadamente los cálculos que podrían haberlo aproximado a una cifra realista en relación con la "masa crítica" con la que funcionaría una bomba, ignoramos si se trató de un error matemático o de una renuncia expresa a tratar de hacer el arma de marras. ${ }^{25}$ Así pues, y como sucede en la mecánica cuántica, la incertidumbre siempre permanece, pero ello no quiere decir que el conocimiento no pueda avanzar: se resuelven algunas variables al tiempo que otras se esconden, y lo que permanece es algo tan impreciso como "[...] that final core of uncertainty at the heart of things." (Frayn, 2000, p. 69).

La forma en la que Volpi recrea el episodio concreto de 1941 es otra. En primer lugar, en las entrevistas de Bacon con Heisenberg y Bohr, cuando se toca el tema del encuentro, ambos personajes reaccionan eludiendo referirse a él. Heisenberg declara que la conversación debe tratarse como un asunto personal entre él y Bohr y que fue una charla muy breve, y sólo confiesa grandes generalidades acerca de los temas tratados: la guerra y la física. Admite que luego de esta visita suya a Copenhague perdió todo contacto con quien había sido su colaborador más estrecho durante los años veinte (recuérdese que la acción de En busca de Klingsor se ubica, en esta parte de la novela, antes de 1947, año en que hubo otro encuentro entre ellos) y, lo que es más significativo, está tembloroso al hablar de todo esto. Queda claro que hubo más en el encuentro que una charla meramente personal; Bacon recurre a Bohr para procurarse más información, pero tampoco este quiere hablar. Cuando le tocan el tema, pretexta que no recuerda mayor cosa. Admite haber hablado con Heisenberg sintiendo una gran incomodidad porque este se hallaba a cargo del proyecto atómico alemán, lo que lo hacía sospechoso, y da a entender que no supo cuáles eran las verdaderas intenciones de Heisenberg durante esa visita: si convertirlo a él en colaborador del proyecto nazi o algo "aún más extraño". En procura de desentrañar lo que pudo ser "aún más extraño" en el encuentro, el texto nos presenta la reconstrucción del narrador (Links) al respecto, pero sin aclarar cuáles son las fuentes en que se basa. Este procedimiento es usual en la novela, y ha sido asumido explícitamente desde el principio: Links sabe todo lo que puede saber como testigo y participante directo de los acontecimientos, todo lo que Bacon le cuenta y todo lo que ha podido reconstruir por otras fuentes, que no se detallan: entendemos que se trata de investigación posterior, sea verosímil o no que haya podido tener acceso a esta. Para decirlo sin ambages, el texto pasa por alto el explicar verosímilmente cómo ha podido Links obtener la información que sólo las pesquisas históricas que realizó Jorge Volpi habría podido proporcionarle. 
La versión de Links establece que Heisenberg es el gestor del encuentro en Copenhague tras muchos esfuerzos, pues su visita no se consideraba especialmente conveniente para el régimen nazi. Detalla lo que fueron las intervenciones públicas o semipúblicas de Heisenberg en Copenhague, ciertamente nada afortunadas para reanimar la amistad entre él y Bohr, pero agrega que este, pese a la oposición de Margrethe, decide invitar a su casa a su viejo amigo. En lo que concierne propiamente a lo que ocurre entonces, Links establece que hay una suspicacia irremediable de parte de Margrethe, que mira a Heisenberg sin nada del aprecio que antes pudo tenerle, y que, cuando los dos hombres hablan solos, lo hacen "[...] como si la propia esencia del lenguaje les impidiese comunicarse [...]" (Volpi, 2000, p. 322). Es Bohr quien más desconfía del otro, el cual le plantea una serie de interrogantes que, al fin y al cabo, se traducen en cuánto de colaborador tiene Heisenberg hacia el régimen nazi y qué es lo que pretende ahora de él. La charla a solas no tiene un buen comienzo, pues Heisenberg se muestra demasiado concesivo con Hitler, al que considera algo así como el mal menor frente a la amenaza de Stalin, y Bohr se enfurece. Pero, antes de que pueda manifestar su ira, Heisenberg lanza la pregunta (la misma que aparece en la versión de Frayn, lo que nos indica que aquí este y Volpi se atuvieron a la misma fuente histórica): “En cuanto físicos, tenemos el derecho moral de trabajar en el desarrollo de la energía atómica?" (Volpi, 2000, p. 323). Bohr reacciona con temor sumado a su enojo, pues ahora no sabe si Heisenberg está hablando de un proyecto que produciría una bomba o un reactor. En esto, la versión de Frayn es coincidente sólo hasta cierto punto, pues en ella se establece claramente que Heisenberg se refería a un reactor, como fuente de energía, y no a una bomba, pero Bohr no supo entonces, en 1941, que así era, y permaneció con dudas. Las siguientes intervenciones de Heisenberg en la versión de Volpi sólo obtienen por respuesta la mudez de Bohr, y todas se organizan en torno a una propuesta: unir la actividad de los físicos en un solo frente de mayor responsabilidad ante las exigencias de los políticos y militares. Se trata de los físicos de uno y otro bando, que podrían llegar al acuerdo de no entregarse a la construcción de una bomba atómica, por encima de las peticiones de aquellos. Esta, al menos, es la interpretación a la que en un inicio se inclina Bacon:26 "Si esta opción fuese correcta, le ofrecía a Bohr detener sus propias investigaciones siempre y cuando los aliados se comprometiesen a hacer lo mismo." (Volpi, 2000, p. 325). La idea de Heisenberg, de haber sido esta (y nótese que el propio Bacon la sigue considerando "una opción" nada más), no podía ser más ingenua, pues ofrecía problemas para su realización que enseguida se hacen explícitos: “¿Cómo podía estar seguro de que Bohr iba a creerle? Y, peor todavía, ¿de que en realidad Bohr fuese a convencer a los Aliados de detener sus investigaciones? ¿Y si Bohr le decía que iba a intentarlo y luego lo traicionaba?” (Volpi, 2000, p. 325). Debido a estos problemas, Bacon considera luego como mayor la posibilidad de que Heisenberg haya sido nada más que un espía enviado por los nazis (en los términos de la trama novelesca, esto equivale a la posibilidad de que Heisenberg haya sido "Klingsor"), y termina por concluir que no hay forma de saber si lo era o no. El episodio termina a oscuras en lo que compete a las intenciones de Heisenberg y a su papel en el trabajo atómico de los alemanes; no así en cuanto a Bohr, de quien se indica que no supo cómo reaccionar ante Heisenberg precisamente por tener esta misma duda en cuanto a sus intenciones.

\section{La bomba atómica}

La historia de la creación de la bomba atómica recibe capítulo aparte en la novela En busca de Klingsor (Volpi, 2000, pp. 380 ss). Volpi recrea tanto la experiencia de los Aliados como la de los nazis en procura de conseguir el armamento que pudo decidir la suerte de la 
Segunda Guerra Mundial. Aunque el narrador sigue siendo en principio Links, es destacable que este es uno de los pasajes en que el texto borra más los rastros de una focalización en torno a la perspectiva del narrador. Por su parte, Copenhague más bien "difumina" la información acerca de la bomba, y aborda con mucho más detalle el lado alemán, dado que Heisenberg tuvo participación en este, mientras que Bohr no llegó al proyecto aliado más que de manera tardía. No obstante, aquí hay una primera diferencia de fondo: Volpi sí le da a Bohr un papel teórico importante en la difusión de la novedad física que permitirá el desarrollo de bombas atómicas: la fisión nuclear, que en esta versión encuentra en el danés a su divulgador principal en los Estados Unidos. Pero luego ambos textos concuerdan en que la participación de Bohr propiamente en la creación de la bomba fue limitada.

El contraste mayor tiene que ver con la participación de Heisenberg. Sin dejar de presentar algún grado de ambigüedad, en Copenhague se exculpa a Heisenberg. ${ }^{27}$ Son Bohr y Margrethe quienes, en el transcurso de los diálogos con este, van descubriendo hechos que terminan por hacernos ver que Heisenberg no quiso colaborar con la creación de una bomba atómica puesta en manos de Hitler. Más bien se plantea que él en primer lugar se enfocó en la construcción de un reactor, un aparato que generaría energía pero no iba a producir bombas. Además, se establece que Heisenberg quiso seguir a cargo de programas de investigación nuclear durante el periodo nazi porque así podía asegurarse de que estos no desembocasen en la construcción de bombas: incluso Heisenberg afirma (Bohr parece creerle; Margrethe tiene sus reservas) que la única razón por la que siguió tomando decisiones era porque así se aseguraba de que no lo sustituyeran por algún otro científico, quizás de menor capacidad pero con muchas más ganas de hacer una bomba: Diebner. Según Heisenberg, pudo "bajarle el perfil" al interés del gobierno nazi por invertir en la investigación nuclear, de manera que los alemanes no pudieran obtener la bomba. Llegó a arriesgar el pellejo, al omitir informaciones ante sus superiores, como el hecho de que el reactor iba a producir plutonio, lo que finalmente posibilitaría las bombas. Y cuando se trató de que quizás, como Margrethe le hace ver, Heisenberg no contribuyó a la creación de la bomba porque estaba equivocado en muchos aspectos técnicos (opinión que Margrethe recoge de Goudsmit, otro científico), Heisenberg defiende el punto de que él dio las explicaciones teóricas convenientes acerca de cómo crear la bomba una vez que los Aliados los habían capturado a él y a los demás participantes de la investigación, y asegura que todo ha quedado grabado en los micrófonos espías con que los vigilaron durante su cautiverio, lo que prueba que él, voluntariamente, estaba saboteando la investigación de los nazis. ${ }^{28}$ Admite un único punto en el que estaba verdaderamente equivocado: el cálculo de la masa crítica, pero luego hace ver que este cálculo él ni siquiera lo realizó precisamente porque no estaba procurando construir una bomba. En conclusión, aunque no deja de haber lugar para ciertas suspicacias como las que plantea Margrethe, en Copenhague tiene mayor fuerza la idea de que Heisenberg era inocente de colaborar genuinamente con el proyecto de la bomba nazi.

Las sospechas contra Heisenberg son mucho mayores en el texto de Volpi. No debe olvidarse que, al fin y al cabo, la novela se acaba sin despejar la incógnita de si Heisenberg no sería el fatídico (aunque ficticio) "Klingsor", malévolo cerebro de la ciencia nazi. En esta versión, se le cobra a Heisenberg que, aunque fue cercano al grupo de los conspiradores que atentaron contra la vida de Hitler (los miembros del Círculo de los Miércoles), se rehusó a participar en el intento de asesinato, y luego, por juzgarse que su lealtad al Reich se hallaba fuera de toda duda, nadie pensó en investigarlo ni mucho menos en detenerlo. Heisenberg era 
un científico de fiar para el régimen nazi, y aunque en un principio tuvo enemigos dentro de este, finalmente conoció un ascenso: "A partir de 1942, la influencia de Heisenberg en política atómica nazi será cada vez mayor" (Volpi, 2000, p. 391). Así, se plantea que sus errores (el que ya hemos comentado en particular: el cálculo de la masa crítica) no provinieron ni de la falta de interés ni mucho menos del deseo de alejar a los nazis de la bomba atómica. Aquí se trata de que genuinamente Heisenberg pensó que sería imposible crear una bomba en el corto plazo (antes de que terminara la guerra) y, al ser transparente en este punto con sus superiores del gobierno, no recibió demasiado apoyo para la investigación. También se trata de que Heisenberg se ve superado experimentalmente por Diebner, indudablemente un científico pro-nazi, pero no porque estuviera tratando de bajarle el perfil a su propia investigación. Finalmente, el texto pone su énfasis en que el grupo de científicos alemanes que continuaron con Heisenberg iban en procura de un reactor por un afán de demostrar alguna superioridad alemana frente a los Aliados, aunque se sabían ya vencidos.

Quizás la mayor duda que la versión de Volpi plantea sea el que Heisenberg fuese invitado a participar en el Círculo de los Miércoles en 1942, un grupo que cultiva un "[...] soterrado y silencioso odio hacia los nazis [...]" (Volpi, 2000, p. 389) y eso no concuerda con la aparente actitud colaboracionista del científico hacia el Reich, precisamente mayor a partir de ese año. Esto sí queda explicado en la versión de Frayn, según la cual Heisenberg nunca fue un genuino colaboracionista nazi, sino tan sólo un patriota preocupado por el destino de Alemania.

\section{Conclusiones}

Los personajes chocan; los textos también. Volpi nos presenta un choque de menor intensidad entre Heisenberg y Bohr, ocurrido solamente a partir de 1941, y resultado no tanto de diferencias teóricas o filosóficas, que en el fondo no tuvieron, como de opciones políticas: Heisenberg coquetea con el nazismo de una forma intolerable para Bohr. La sombra de Klingsor se yergue sobre el Heisenberg de la novela: nunca sabremos cuán cercano se mantuvo respecto al régimen nazi, si fue un genuino partidario de los nazis o solamente un colaboracionista por conveniencia o hasta por cobardía. Pero lo que sí sabemos es que estuvo muy lejos de ser, en esta versión, el posible mediador para la paz que Frayn plantea. La posibilidad de que Heisenberg haya querido una especie de "tratado de paz" entre nazis y aliados durante su visita de 1941 a Copenhague se plantea en Volpi como muy remota. Por el contrario, esta es la posibilidad que resulta más defendible en la versión de Frayn: Heisenberg, el patriota que no puede querer la destrucción de su patria pero tampoco desea plegarse a Hitler, parece haber planeado la resistencia al nazismo tanto como haber procurado la preservación de Alemania. ${ }^{29}$

Ahora bien, en ambas versiones permanece una cierta incertidumbre al respecto de los papeles que cumplieron los personajes, algo que no se puede aclarar por completo. Menor quizás en el caso de Bohr, algo en lo que coinciden los dos textos. Bohr nunca parece un candidato al puesto de Klingsor; por otro lado, en Copenhague jamás se duda de sus buenas intenciones durante la guerra, si bien quizás estas lo pudieron llevar a colaborar en una pequeña medida con los creadores de la bomba atómica. El choque entre Bohr y Heisenberg es mucho mayor según Frayn, ya que comienza con una serie de diferencias teóricas en relación con temas de fondo: la incertidumbre, la complementariedad y la misma interpretación de Copenhague, y de ahí se extiende a una relación personal básicamente conflictiva, aunque no carente de afectividad; en una palabra, compleja, como la de un padre y un hijo. 
La versión de Frayn acerca del principio antrópico, débil o fuerte, es mucho más fuerte, valga la redundancia. En la obra se subraya la posibilidad de que la realidad física sea la consecuencia de la observación por parte de los seres humanos, si bien se la plantea como una posibilidad un tanto especulativa. La versión de Volpi al respecto del principio antrópico se limita a ser una extensión de la conciencia de un cierto perspectivismo literario, que resalta la subjetividad intrínseca del lenguaje.

Las lecturas de los aspectos científicos son también, incluidos los principios antrópicos, parcialmente contrastantes. Sin repasar los detalles que ya se expusieron, quiero destacar que, en este caso, las versiones literarias se diferencian en sus énfasis primeramente y, segundo, en los alcances filosóficos que leen en tales aspectos científicos. Pero más notable aún me parece el hecho de que los textos literarios aquí no sean radicalmente diferentes de los textos escritos por científicos, que también, en lo que a mecánica cuántica respecta, difieren en cierta medida, sobre todo en lo segundo. La mecánica cuántica ha creado un saber científico sobre cuyas repercusiones filosóficas no existe consenso. Por otro lado, queda asentado sobre el saber científico que esté también está mediado por intereses y consideraciones políticos y personales, que alejan a sus practicantes de la "pureza" o la "objetividad" en los sentidos más ingenuos que suelen concedérseles a estas supuestas características de la ciencia.

Por ello, mi conclusión más general es que, si los personajes chocan y los textos chocan, es porque los saberes chocan. Pero ello no debe desencantarnos en nuestra búsqueda del conocimiento, y mucho menos detenernos. Pues, como ocurre con los experimentos que tienen lugar en el Gran Colisionador de Hadrones, conseguimos asomarnos parcialmente al conocimiento al observar los efectos de los choques.

\section{Notas}

1. Se trata de dos textos literarios que no pasan por alto los pormenores del quehacer científico de estos personajes.

2. En la postdata de su novela, Volpi no tiene problemas en comenzar por admitir que su obra literaria es ficcional (lo que no quiere decir, siguiendo a Eco, que sea falsa). Otra parece ser la actitud de Michael Frayn, muy preocupado por dotar de exactitud histórica la versión de los hechos que presenta: una gran cantidad de páginas de su "Postscriptum" se dedican a justificar su obra sobre la base de la investigación llevada a cabo, e incluso a polemizar con otras versiones presentadas no en obras literarias sino en textos de carácter histórico.

3. Su nombre propiamente es Gran Colisionador de Hadrones; "Large Hadron Collider", en inglés. Si bien no hay demasiada necesidad de recordar este idioma ya que los estadounidenses no construyeron este aparato. Los hadrones son partículas subatómicas, pero no se las postula tan elementales que no estén ellas mismas formadas por otras partículas, los quarks. En este aparato, los haces de partículas son acelerados progresivamente hasta alcanzar velocidades cercanas a la de la luz; se envían en sentidos opuestos de manera que colisionen, todo ello con propósitos experimentales.

4. $\quad$ En el acto segundo, viene este diálogo: "Heisenberg: Meanwhile you were going on from Sweden to Los Alamos. / Bohr: To play my small but helpful part in the deaths of a hundred thousand people. / Margrethe: Niels, you did nothing wrong! / Bohr: Didn't I?” (Frayn, 2000, p. 67) Así se plantea la duda, ya muy cerca del final: ¿Quién es más culpable de ellos dos?

5. En una película reciente de Quentin Tarantino, Inglorious Bastards, se narra la forma cómo Hitler y toda su plana mayor fueron muertos en un cine durante la ocupación de Francia. El espectador se sorprende solamente un momento, antes de decidir que lo han estafado al suponerlo capaz de creer algo así. Por eso es de justicia reconocer que Frayn, aunque excedido en su fe de que es posible hacerse con la verdad mediante la obra literaria, tiene el mérito de no proponer que la ficción puede simplemente dejarse 
llevar por la arbitrariedad fantasiosa, sobre todo cuando trata con personajes cuyo referente histórico forma parte del saber de los lectores. Me temo que es lo que está implicado en el filme de Tarantino, en mi opinión: un soberano desprecio de la cultura del espectador, a quien al menos habría debido proponérsele alguna hipótesis acerca de lo que habría pasado si Hitler hubiera muerto en Francia. Todo lo contrario: Tarantino termina sin ir más allá.

6. Sería posible profundizar en la investigación de las relaciones que el texto permite establecer entre el Francis Bacon histórico y el Francis Bacon ficcional, pero ello no es mi objetivo ahora. Aquí se abren un sinfín de posibilidades, tomando en cuenta la vida llena de vicisitudes que tuvo Bacon en la Inglaterra isabelina, así como los misterios que aún hoy rodean su figura. No olvidemos la sospecha tradicional de que este se ha llegado a considerar "culpable" de algo muy importante para las letras: la autoría de las obras de Shakespeare. Eso sin mencionar su relación enigmática con grupos religiosos, su controvertida orientación sexual, y por supuesto el detalle de sus aportes propiamente dichos a los quehaceres científicos.

7. La función de onda es una representación matemática compleja del estado de una partícula o sistema de partículas.

8. Según parece, Schrödinger se vio obligado a guardar cama, con fiebre, durante sus "amigables" conversaciones con Bohr.

9. Incluso se habla de que al jugar ping pong la expresión de Bohr era amenazante, si bien él no se daba cuenta.

10. De aquí surgen las diferencias entre la mecánica matricial y la mecánica ondulatoria.

11. Aquí Volpi también relata el episodio en el que Bohr ha invitado a Schrödinger para discutir sobre la mecánica ondulatoria y la mecánica matricial y este último incluso cae enfermo ante las muchas presiones de Bohr.

12. Se refiere a cómo se ve a un fotón.

13. Volveré sobre esto. En realidad, Volpi lee como "complementariedad" lo que Frayn y otros consideran que es propiamente la interpretación de Copenhague.

14. Sobre la dificultad propia del pensamiento de Bohr, también Martin Rees comenta: "Niels Bohr [...] recomendaba a sus colegas "hablar tan claro como se piensa, pero no más". Ciertamente, él mismo tendría que haber seguido su propio consejo, pues era famoso por la inaudibilidad y la oscuridad de su discurso." (Rees, 2001, p. 15).

15. Si bien, en la exposición de estos autores, ellos la presentan como el primer pilar, antes de la incertidumbre y la complementariedad: véase pp. 126-ss.

16. Para hacer justicia a Martin Rees, hay que agregar que en otra parte expresa algunas dudas acerca de lo que acaba de citarse: "El principio antrópico fuerte de Carter es más controvertido y especulativo: afirma que las leyes fundamentales de cualquier universo deben ser tales que permitan la existencia de observadores." (Rees, 2001, p. 262) "El "universo participativo” parece difícil de aceptar, y hasta de tomar en serio. ¿Qué suerte de observador hay que invocar para "dar existencia al universo”? ¿Un ratón? ¿Un ser humano? ¿Un doctor en física?” (Rees, 2001, p. 263).

17. Y esta vez no parece que Wheeler exprese ningún género de dudas al respecto.

18. Consúltense las páginas 110 a 122 , si se quiere conocer el detalle del experimento. No lo resumo aquí pues su complejidad implicaría el dedicarle muchas páginas. Pero sí recomiendo su lectura, dado que además se ha planteado como una demostración hecha ante un grupo de personas cultas y desprejuiciadas, y no necesariamente con conocimientos profundos de física.

19. No Volpi exactamente, sino Links, el narrador ficcional... Pero aquí se manifiesta el juego que enseguida trataré de explicar.

20. Principio antrópico débil.

21. Principio antrópico fuerte.

22. "Todo narrador tiene un motivo para narrar", la Ley III, no aporta nada nuevo: tan sólo es otra expresión de subjetivismo.

23. Pareciera haber dos grandes tendencias de los físicos ante la mecánica cuántica o, al menos, eso es que he podido detectar en este aspecto: los que la ven convencidos de que Bohr tenía razón y es muy extraña 
(Rosenblum y Kuttner [2011] por ejemplo) y los que creen que tal extrañeza debe y puede superarse, con sólo alguna profundización en el conocimiento que haga ver como perfectamente normales sus supuestas fuentes de asombro (Gell-Mann, por ejemplo).

24. Así lo plantea Shepherd-Barr (2006, p. 91).

25. Lo ignoramos inicialmente: luego queda claro que fue lo segundo.

26. Tampoco aquí queda claro cómo pudo saber Bacon en ese momento lo que Links acaba de narrar. Es, como he dicho, un elemento de la verosimilitud del texto que se pasa por alto.

27. El "Postscriptum" lo hace ya sin ninguna ambigüedad, lo cual no deja de ser un tanto decepcionante. Uno siente demasiada intervención por parte del autor.

28. En el "Postscriptum", Frayn declara que lo que inclinó la balanza hacia su decisión de exculpar a Heisenberg, entre tantas opiniones diversas, fue su conocimiento de las grabaciones (secretas hasta 1992) que tuvieron los científicos alemanes capturados, durante su confinamiento en Inglaterra.

29. El "Postscriptum" de Michael Frayn se encarga de justificar ampliamente, sobre la base de la documentación histórica, esta versión, tal como indiqué previamente.

\section{Bibliografía}

Barthes, R. (1987). S/Z. (N. Rosa, tr.). México, D.F.: Siglo Veintiuno.

Bohr, N. (1987). The Philosophical Writings of Niels Bohr. Connecticut: Oxbow Press.

Brockman, J. (Ed.). (2000). La tercera cultura. Más allá de la revolución científica. (A. García, tr.). Barcelona: Tusquets.

Chimal, C. (2000). Luz interior. Conversaciones sobre ciencia y literatura. Barcelona: Tusquets.

Dawkins, R. (2012). Destejiendo el arco iris. (J. Ros, tr.). Barcelona: Tusquets.

Eco, U. (1981). Lector in Fabula. (R. Pochtar, tr.). Barcelona: Lumen.

Frayn, M. (2000). Copenhagen. New York: Anchor Books.

Gell-Mann, M. (2007). El quark y el jaguar. Aventuras en lo simple y lo complejo. (A. García y R. Pastor, tr.). Barcelona: Tusquets.

Hawking, S. y Mlodinow, L. (2010). El gran diseño. ( D. Jou i Mirabent, tr.). Barcelona: Crítica. Heisenberg, W. (1958). Physics and Philosophy. New York: Penguin.

Ledermann, L.M. y T. Hill, C. (2006). La simetría y la belleza del universo. (L.E. de Juan, tr.). Barcelona: Tusquets.

Lewin, R. (2002). Complejidad. El caos como generador de orden. (J.G. López-Guix, tr.). Barcelona: Tusquets.

Penrose, R. (2012). Las sombras de la mente. Hacia una comprensión científica de la conciencia. (J. García-Sanz, tr.). Barcelona: Crítica.

Rees, M. (2001). Antes del principio. El cosmos y otros universos. (N. Herrán, tr.). Barcelona: Tusquets.

Rosenblum, B. y Kuttner, F. (2011). El enigma cuántico. Encuentros entre la física y la conciencia. (A. García, tr.). Barcelona: Tusquets.

Schrödinger, E. (1947). What is life? Cambridge: Cambridge University Press. 
Shepherd-Barr, K. (2006). Science on stage. From Doctor Faustus to Copenhagen. Princeton y Oxford: Princeton University Press.

Vega, M.J. y Carbonell, N. (1998). La literatura comparada: principios y métodos. Madrid: Gredos.

Volpi, J. (2000). En busca de Klingsor. Barcelona: Seix Barral. 
